\title{
国家微重力实验室百米落塔实验 设施的几项关键技术
}

\author{
张孝谦 ${ }^{\circledR} \quad$ 袁龙根 $^{\circledR}$ 吴文东 ${ }^{\oplus}$ 田兰桥 $^{\circledR}$ 姚康庄 $^{\circledR}$
}

(1) 中国科学院工程热物理研究所, 北京 100080; (2) 中国科学院力学研究所, 北京 100080)

\begin{abstract}
摘要在国家 863 计划的支持和安排下于 2003 年建成的国家微重力实验室百 米落塔是我国发展载人航天, 开发利用外层空间计划中进行微重力科学实验的 重要地基设施. 与美、德、日等国的同类设施相比, 我国的这座落塔在技术性能 上先进水平, 并具有我国自己的特色. 其主要组成部分: 实验舱组件、减速回收 系统和释放系统都展现了其先进性和原始创新性, 显示了我国在这个领域内的 实力.
\end{abstract}

\section{关键词落塔实验设施 实验舱组件 减速回收系统 释放系统}

发展载人航天，建立空间站，开发利用外层空间，探索深层空间等空间计划是 我国 21 世纪初期科技发展的重要战略目标之一. 微重力科学与应用是这项计划的 重要组成部分. 国际上随着载人航天和开发利用外层空间计划的实施, 微重力科 学与应用领域的研究, 发展工作一直十分活跃, 并已取得很多重要成果. 国际空间 站的即将建成与投入使用必将进一步推动国际上在这个领域的研究发展工作.

为了实现我国空间计划的战略目标，在国家 863 计划的支持和安排下于 20 世纪 90 年代中期开始组建国家微重力实验室(NMLC). 百米落塔实验设施是国家 微重力实验室的主要设施之一. 图 1 给出了落塔的外观景象. 塔高 $116 \mathrm{~m}$, 座落在 中关村核心区东南部分, 是中关村核心区的最高建筑, 它将承担繁忙的微重力科 学实验研究任务.

这座科学研究型的百米落塔, 将在国际上对外开放, 所以它既要满足我国空 间计划的需要, 又要在技术性能上达到国际先进水平, 以显示我国科学技术的实 力. 但是在经费投资上却远远低于美、德、日等国的同类设施的经费投资. 因而,

2004-08-16 收稿, 2005-02-13 收修改稿

SCIENCE IN CHINA Ser. E Engineering \& Materials Science 


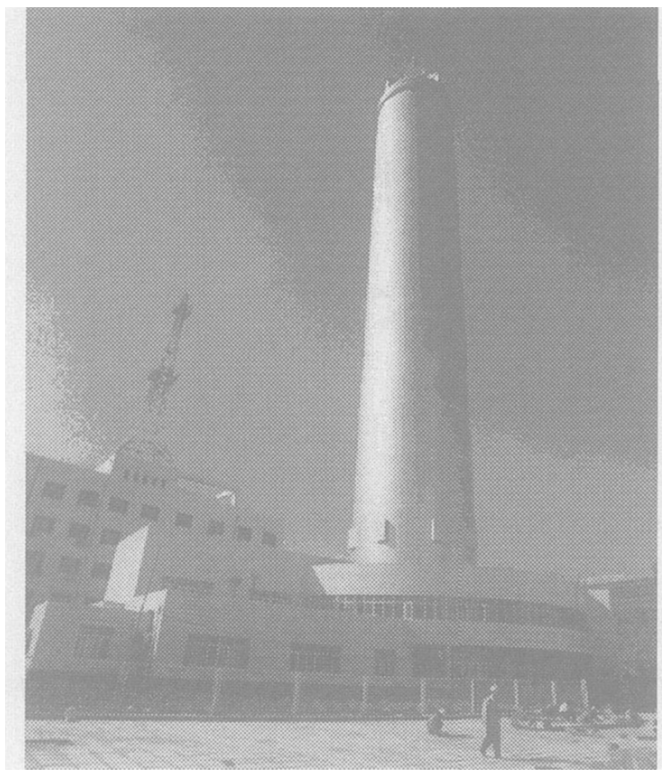

图 1 落塔的外观景象

在建立这座落塔的过程中，在借鉴国 际上的经验和教训的同时, 要开创出 自己的特色. 在这座落塔设施的主要 组成部分: 实验舱组件、减速回收系统 和释放系统上都展现了其先进性和原 始创新性. 本文将简要地介绍这几项 关键技术的特点和原始创新性.

\section{1 国际上主要微重力落塔(井) 实验设施概况}

进行微重力科学实验有多种途径, 常用的有落塔(井)、做抛物线飞行的飞 机、探空火箭、空间实验室、航天飞机 以及空间站等设施(图 2) ${ }^{[1]}$. 这些设施 各有特点和应用范围，彼此不能取代，相互补充，组成一套较为完整的体系，能 够承担各种类型的实验任务.

与其他各种设施相比，落塔(井)虽然有微重力时间较短的不足之处，但是却 具有微重力水平高, 费用低, 实验机会多, 使用频率高(每天两次或更多次实验), 能够使用较精密的测量仪器, 便于人为干预等明显的优点, 使得落塔(井)成为进 行微重力科学实验的最主要的设施.

早在 20 世纪 60 年代, 随着美国载人航天计划的实施, NASA 在 Lewis 研究中 心(Lewis Research Center, 简称 LeRC, 现改称 Glenn 研究中心)相继建立了两座落 塔 $(\text { 井 })^{[1 \sim 3]}$. 一座 “零重力研究设施” 是大型的落井设施, 深入地下 $155 \mathrm{~m}$, 能够提 供 $5.18 \mathrm{~s}$ 微重力时间, 微重力水平为 $10^{-5} \sim 10^{-6} g_{0}$, 平均减速过载为 $35 g_{0}$, 峰值过 载 $65 \mathrm{~g}_{0}$. 另一座落塔是简易型的设施, 塔高 $30.5 \mathrm{~m}$, 能提供 $2.2 \mathrm{~s}$ 微重力时间, 微 重力水平为 $10^{-4} g_{0}$, 减速过载为 $15 \sim 30 g_{0}$, 这座落塔承担了大量的实验任务, 平时 每天进行 6 8 次实验, 实验任务繁忙时每天可进行 12 次实验.

随着世界范围内空间计划和微重力科学与应用的快速发展，日本于 20 世纪 80 年代末, 90 年代初相继建立了两座微重力实验设施. 一座是世界上最大的微重 力落井设施 ${ }^{[4,5]}(\mathrm{JAMIC})$, 深入地下 $710 \mathrm{~m}$, 能提供 $10 \mathrm{~s}$ 微重力时间, 微重力水平 为 $10^{-5} g_{0}$, 减速过载小于 $10 g_{0}$. 另一座微重力落井设施 $[6,7]$ 深入地下 $150 \mathrm{~m}$, 能提 供 $4.5 \mathrm{~s}$ 微重力时间, 微重力水平为 $10^{-5} g_{0}$, 减速过载小于 $10 g_{0}$.

德国也于 20 世纪 80 年代末, 90 年代初在 Bremen 建立了一座落塔 ${ }^{[8,9]}$. 塔高 $146 \mathrm{~m}$, 能提供 $4.7 \mathrm{~s}$ 微重力时间, 微重力水平为 $10^{-5} \sim 10^{-6} g_{0}$, 平均减速过载 $25 g_{0}$, 峰值过载 $50 g_{0}$. 


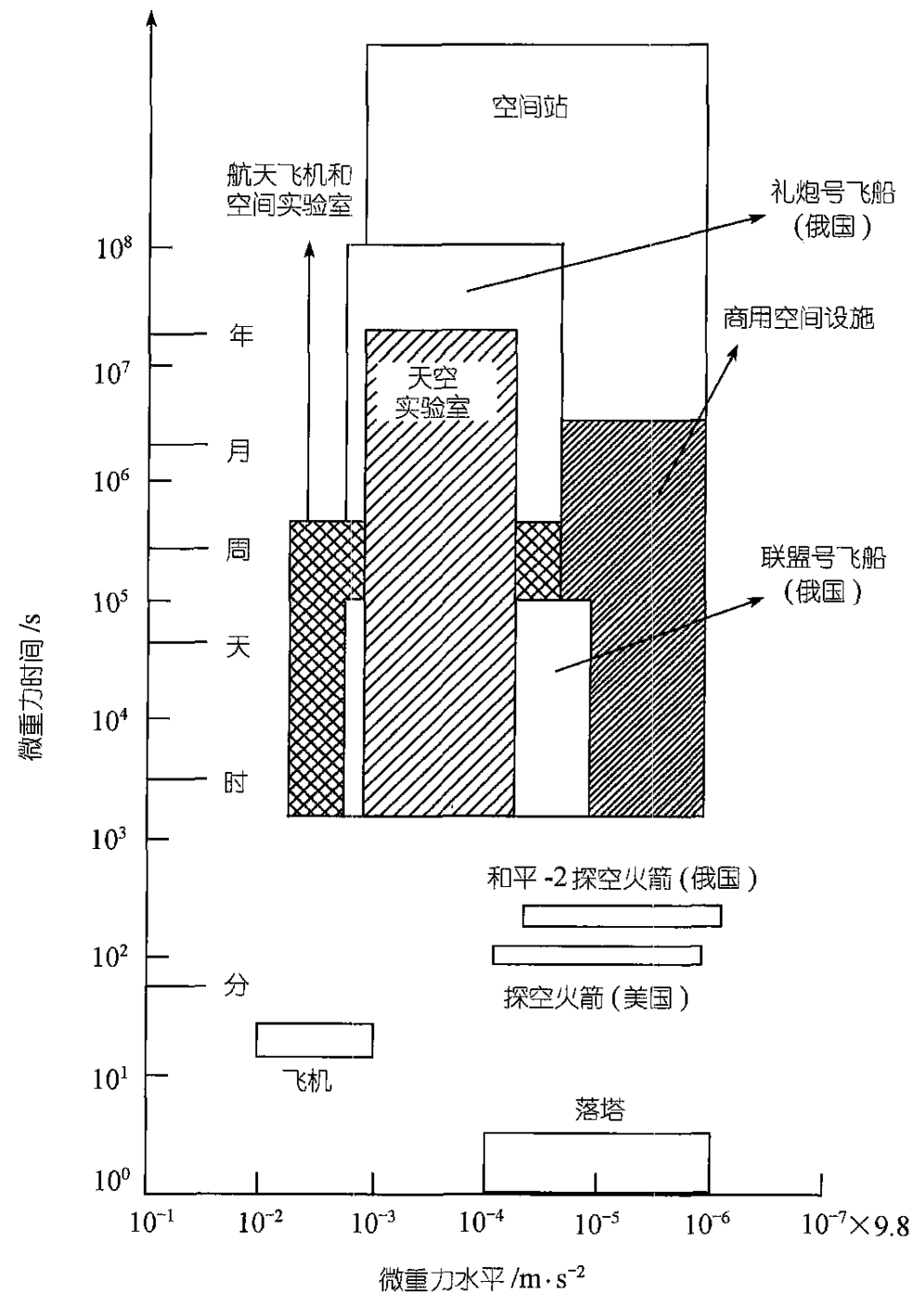

图 2 微重力实验设施特征时间和微重力水平

据了解，俄罗斯(前苏联)在发展空间计划时也建立了大型落塔(井)设施，但 未见公开资料报导. 此外, 美国的一些大学和研究院(所)内还有多座微重力时间 $\leqslant 1 \mathrm{~s}$ 的小型微重力实验设施.

\section{2 国家微重力实验室百米落塔实验设施}

百米落塔自由落体实验设施的主要技术性能为微重力时间: $3.5 \mathrm{~s}$; 微重力水 平: $10^{-5} g_{0}$; 减速过载: 近似半正弦波平均: $\sim 8 g_{0}$, 峰值: $\sim 12 g_{0}$.

这套设施由实验舱组件、减速回收系统、释放系统、控制系统、测量系统以 及辅助设施组成. 本文仅概要地介绍其中技术上先进并具有原始创新性的实验 舱组件、减速回收系统和释放系统. 


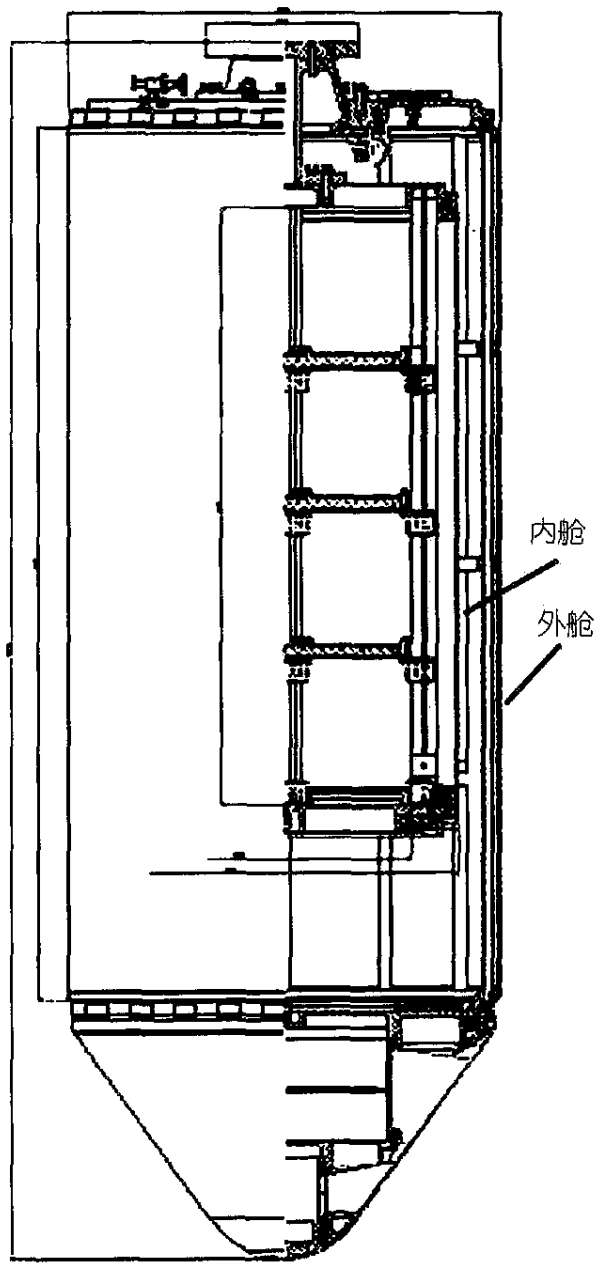

图 3 实验舱结构

\section{1 实验舱组件}

实验舱为内外舱间抽真空的双层套 舱结构(图 3). 实验时外舱在大气中下落. 设计时选取了轴对称的气动外 形, 以尽 量减小下落时舱体承受的气动阻力. 在 减速回收过程中实验舱组件要承受较大 的冲击力. 总体方案上要求外舱为轻型、 薄壁、受压组合壳体结构. 设计时经过 详细的受力分析 ${ }^{1), 2)}$ (包括: 设计规范校 核、壳体理论核算、有限元详细计算)和 反复修改完善, 从而确保了舱体有足够 安全的强度、刚度和稳定性. 在设施调试 和试运行过程中, 实验舱组件经受了考 验, 完全满足了使用要求.

内舱中装有实验装置, 实验时内舱 在外舱内真空环境中随外舱一起自由下 落，内外舱间相对速度很小 $(<1 \mathrm{~m} / \mathrm{s})$. 真 空环境和很小的相对速度这两点有效地 减小了内舱自由下落时受到的气动阻力, 从而确保内舱中的实验装置达到 $10^{-5} g_{0}$ 甚至更优的微重力水平.

这种内外舱间抽真空的轻型双层套 舱结构在国际上是首次使用. 与国外同类型设施相比，本实验舱组件具有下列明 显的特点和优越性:

(i) 为了获得 $10^{-5} \sim 10^{-6} g_{0}$ 的微重力水平, 必须确保装有实验装置的舱段在自 由下落的过程中受到的气动阻力足够的小. 自由落体下落时受到的气动阻力通 常表示为 $F=\frac{1}{2} C_{x} s \rho u^{2}$, 其中 $C_{x}$ 为阻力系数, $s$ 为特征截面积, $\rho$ 为舱在介质中下 落时介质的密度, $u$ 为舱在介质中的相对运动速度. 在实验舱的气动外形设计和 结构设计确定后, 阻力系数 $C_{x}$ 和特征截面积 $s$ 随之确定. 要想使气动阻力足够的 小，可以通过降低介质(气体)的密度 $\rho$ 和/或降低舱在介质(气体)中的相对运动速 度 $u$ 的方法. 从原理上分析，降低相对运动速度比降低介质(气体)密度对减小气

1) 田宗漱，高陆. 国家微重力实验室自由落体设施——落舱受力分析. 1998

2) 田宗漱，高陆. 落舱结构改进后的受力分析(强度、刚度、屈曲有限元分析). 1999 
动阻力更为有效. 但是, 从技术上分析, 降低介质(气体)密度却比降低相对运动 速度简易得多.

国际上, 德国 Bremen 落塔和美国 NASA LeRC 的零重力研究设施均采用降 低介质(气体)密度的方法(单舱在真空钢管内下落). 德国 Bremen 落塔的真空钢管 内径 $3.5 \mathrm{~m}$, 高 $110 \mathrm{~m}$, 再加上安置减速回收筒的工作室, 需要抽真空的容积达 $1700 \mathrm{~m}^{3}$. 配置了 18 台真空原组, 抽速为 $32000 \mathrm{~m}^{3} / \mathrm{h}(9000 \mathrm{~L} / \mathrm{s})$, 需抽真空 $2.5 \mathrm{~h}$, 真空钢管内可达 $1 \mathrm{~Pa}$ 的真空度. 这样, 实验舱(单舱)在 $1 \mathrm{~Pa}$ 的真空环境中下落可 以达到 $10^{-5} \sim 10^{-6} g_{0}$ 的微重力水平. 美国 NASA LeRC 零重力研究设施真空钢管的 内径 $6.1 \mathrm{~m}$, 高 $145 \mathrm{~m}$, 配置了更大抽速的真空泉组. 而本设施则同时采用了降低 介质(气体)密度和舱在介质(气体)中相对运动速度的方法, 这样将更有效地减小 实验舱下落时受到的气动阻力. 采用内外舱间抽真空的双层套舱结构, 实验时内 舱也是在真空环境中下落, 只是这个真空环境(外舱)随内舱一起下落. 需要抽真 空的容积(内、外舱间的空间)仅 $1 \mathrm{~m}^{3}$, 只需配置一套抽速为 $8 \mathrm{~L} / \mathrm{s}$ 的小型真空原 组, $20 \mathrm{~min}$ 就可抽到所要求的真空度. 这样不仅大幅度地降低了固定设备的投资 费 用，而且还可节省大量的正常运行和维修费用.

(ii) 德国 Bremen 落塔和美国 NASA LeRC 零重力研究设施实验舱均是单舱 结构, 下落时舱体速度以 $1 g_{0}$ 的加速度迅速增加, 在自由下落终止时实验舱的速 度将分别达到 $46 \mathrm{~m} / \mathrm{s}$ 和 $51 \mathrm{~m} / \mathrm{s}$. 由于实验舱下落时受到的气动阻力与舱的相对 速度的平方成正比, 为了要获得 $10^{-5} \sim 10^{-6} g_{0}$ 的微重力水平, 必须将真空钢管内的 压力抽到较高的真空度 1 10 Pa.

而本设施内、外舱一起下落, 内、外舱的相对速度 $<1 \mathrm{~m} / \mathrm{s}$, 这样将更有效地 减小内舱(实验舱)下落时受到的气动阻力. 数值分析结果表明 ${ }^{1)}$, 由于本设施内、 外舱相对速度很小 $(<1 \mathrm{~m} / \mathrm{s})$, 只要内、外舱间的真空度 $<1000 \mathrm{~Pa}$ 时, 内舱的微重力 水平即可达到 $10^{-5} g_{0}$. 按国际目前通用方法用加速度计实测的数据也证明了这一 结果. 本设施现在实际工作的状态是内、外舱间压力抽到 $<30 \mathrm{~Pa}$. 根据数值分析, 在这种情况下, 内舱的微重力水平可达到 $10^{-6} g_{0}$, 但国内目前尚无合适的加速度 计来实际测量出来.

(iii) 日本 JAMIC 落井的落舱也是双层套舱，内外舱间抽真空 $10 \mathrm{~Pa}$ ，但其使 用的是重型结构, 落舱总质量达 $5000 \mathrm{~kg}$. 而本设施的落舱却是轻型, 薄壁结构, 总质量仅为 $600 \sim 630 \mathrm{~kg}$.

（iv）美国 NASA LeRC $2.2 \mathrm{~s}$ 落塔实验舱也采用双层套舱结构，但内、外舱间 不抽真空. 这样实验舱的力学设计及和舱结构密切相关的释放装置的设计在技 术上就要简单得多. 但由于内、外舱间未抽真空, 内舱中的实验装置的微重力水

1) 中国科学院国家微重力实验室落塔调试组 2000.4-2002.6 部分文档. 2002

www.scichina.com 
平也仅能达到 $10^{-4} \mathrm{~g}_{0}$.

本设施这种轻型双层套舱，内、外舱间抽真空的实验舱结构，具有上述特点 和优越性, 但是在技术上却十分困难. 可能也正是这种原因, 德国 Bremen 落塔和 美国 NASA LeRC 零重力研究设施在经过详细的分析比较和论证后, 还是采用了 耗费巨大的单舱在真空钢管中下落的方案. 本设施的方案在具体实施时, 确实遇 到了一系列很困难的技术问题，经过大量的调试工作，这些问题逐步地一一解决 了.

首先是内舱与外舱自由下落时的姿态问题，这个问题与释放装置的配合密 切相关. 这部分内容将在下面讨论释放系统部分时论述.

第二，内、外舱间径向间距的选取. 首先内舱必须有足够的安装实验装置， 内务数据的测量及记录装置、视频监测装置、舱载计算机装置、无线传输装置以 及电源等有效载荷的空间. 同时, 为了尽量减小外舱下落时受到的气动阻力, 外 舱的特征截面积要力所能及的小. 但是内、外舱在自由下落时由多种不能完全消 除的干扰所引起的下落姿态的侧斜, 可能造成在自由下落过程中内舱提前和外 舱内壁面侧碰, 缩短了微重力时间, 并影响实验舱组件进入减速回收网袋的姿 态 $^{1)}$. 因此, 内外舱间径向既要留有足够的安全间距, 同时此径向间距也要力所 能及的小. 在调试实验中, 我们用高速摄像机(1000 帧/s)拍摄外舱下落姿态, 在 外舱内用 CCD 摄像监测下落时内舱相对于外舱的姿态. 在采取了多项减小干扰 的技术措施之后，有效地控制了内、外舱的下落姿态 ${ }^{2)}$. 现在，内、外舱间径向间 距选为 $10 \mathrm{~cm}$ ，运行结果表明这个径向间距是合适的，满足了使用要求.

第三，内、外舱间轴向距离的选取. 实验时, 外舱在大气中下落受气动阻力 的作用, 下落速度相对慢一些. 内舱是在真空环境中, 以很小的相对速度下落, 受到的气动阻力很小, 因而绝对下落速度就比外舱快. 所以内、外舱间必需留有 足够的轴向距离, 以确保在实验舱进入减速回收过程前内舱不碰到外舱下底板 上. 从减速回收过程的角度来考虑, 这个轴向距离又不能留得太长, 而是要求在 自由下落过程结束时, 内舱正好座落在外舱下底板上, 内、外舱作为一个整体进 入减速回收过程. 这样, 既可以最充分地利用内舱自由下落高度，得到可能得到 的最长的微重力时间, 又能避免在外舱已经减速后, 内舱再落到外舱下底板上而 引起的冲撞造成较大的冲击过载. 调试过程中, 我们采用了轴向距离可调, 并逐步 增加的方法 ${ }^{2)}$. 结果表明这种方法安全可靠, 且行之有效. 现在此距离选为 $81 \mathrm{~cm}$.

第四，内、外舱质心与其几何对称轴的偏心率的控制. 如果舱体的质心不在 其几何对称轴线上，这种质心的偏心会造成在舱体被释放的瞬间受到一个初始

1) 中国科学院国家微重力实验室落塔调试组. 影响落舱自由下落姿态的几种因素. 2003

2) 中国科学院国家微重力实验室落塔调试组. 落舱实验系统调试技术总结报告. 2003

SCIENCE IN CHINA Ser. E Engineering \& Materials Science 
干扰力矩, 从而导致舱体自由下落时侧斜, 所以舱体质心的偏心率必须严格控 制. 德国 Bremen 落塔单舱的质心偏心率限制在 $\leqslant 1 \mathrm{~kg} \cdot \mathrm{m}^{[8]}$. 而我们的落塔采用 的是双层套舱，内、外舱间抽真空的结构. 由于内、外舱间径向间距要力所能及 的小, 所以舱体质心的偏心率就必须限制在更严格的范围内. 通过调试实验, 现 在内、外舱的质心偏心率均控制在 $\leqslant 0.3 \mathrm{~kg} \cdot \mathrm{m}$ 范围内. 运行结果表明这种控制 是必要和合适的.

第五, 外舱质心的位置应尽量靠近舱体底部, 以确保在外舱下落姿态出现侧 斜时，舱体受到的气动阻力的合力和舱体的重力组合的结果起恢复舱体正直姿 态的作用.

第六，当内舱正好坐落在外舱下底板上作为一个整体进入减速回收过程时， 内舱必须稳稳地座落在外舱下底板上, 不易晃动和侧倒, 否则可能导致减速回收 过程的安全故障. 现在在外舱下底板上配置了一个内舱锁紧机构 ${ }^{1)}$, 当内舱刚坐 落在外舱下底板上时锁紧机构立即将其锁住，使其不易晃动和侧倒.

\section{2 减速回收系统}

本设施的减速回收系统使用的是我国独特的弹性可控减速回收装置, 这套 装置由三端互逆机/电换能器(以下简称换能器)、钢丝绳索、钢质圆环、高强度编 织网袋(以下简称网袋)、高强度弹性橡皮筋绳组(以下简称橡皮筋绳组)、耗能电 阻箱、工控机控制单元以及紧急制动筒等组成(图 4). 这套装置的工作过程及原 理为

(i) 换能器(此时, 换能器为电动机功能)将钢质圆环、橡皮筋绳组和网袋 (网 袋组件)提升到预定高度并悬停处于待命状态.

(ii) 当落舱下落接触网袋前 $1 \mathrm{~s}$, 在工控机指令下网袋组件开始在自重作用 下下滑, 带动换能器, 此时换能器为发电机功能, 换能器转动时发出的电由耗能 电阻箱耗掉. 这样, 在自由下落的落舱组件落入网袋时换能器已经处于转动状 态，以减小换能器受到的冲击.

(iii) 当质量为 $600 \sim 630 \mathrm{~kg}$ 的落舱组件, 以 $34 \mathrm{~m} / \mathrm{s}$ 速度落入网袋组件后, 落 舱组件减速，而网袋组件加速下落，同时橡皮筋绳组被拉长，将落舱组件的动能 转换为弹性势能暂时储存. 此时, 在网袋组件加速下落拖动下, 换能器加快转动, 在工控机的指令下将部分落舱组件的动能转换为电能, 并由耗能电阻箱消耗掉.

(iv) 当换能器转速的折合线速度超过网袋组件下落速度时, 落舱组件和网 袋组件在继续下落的同时，橡皮筋绳组开始收缩，释放出所储存的弹性势能，通 过换能器转换为电能由耗能电阻箱消耗掉. 此时落舱组件进一步减速.

1) 中国科学院国家微重力实验室落塔调试组. 落舱实验系统调试技术总结报告. 2003

www.scichina.com 


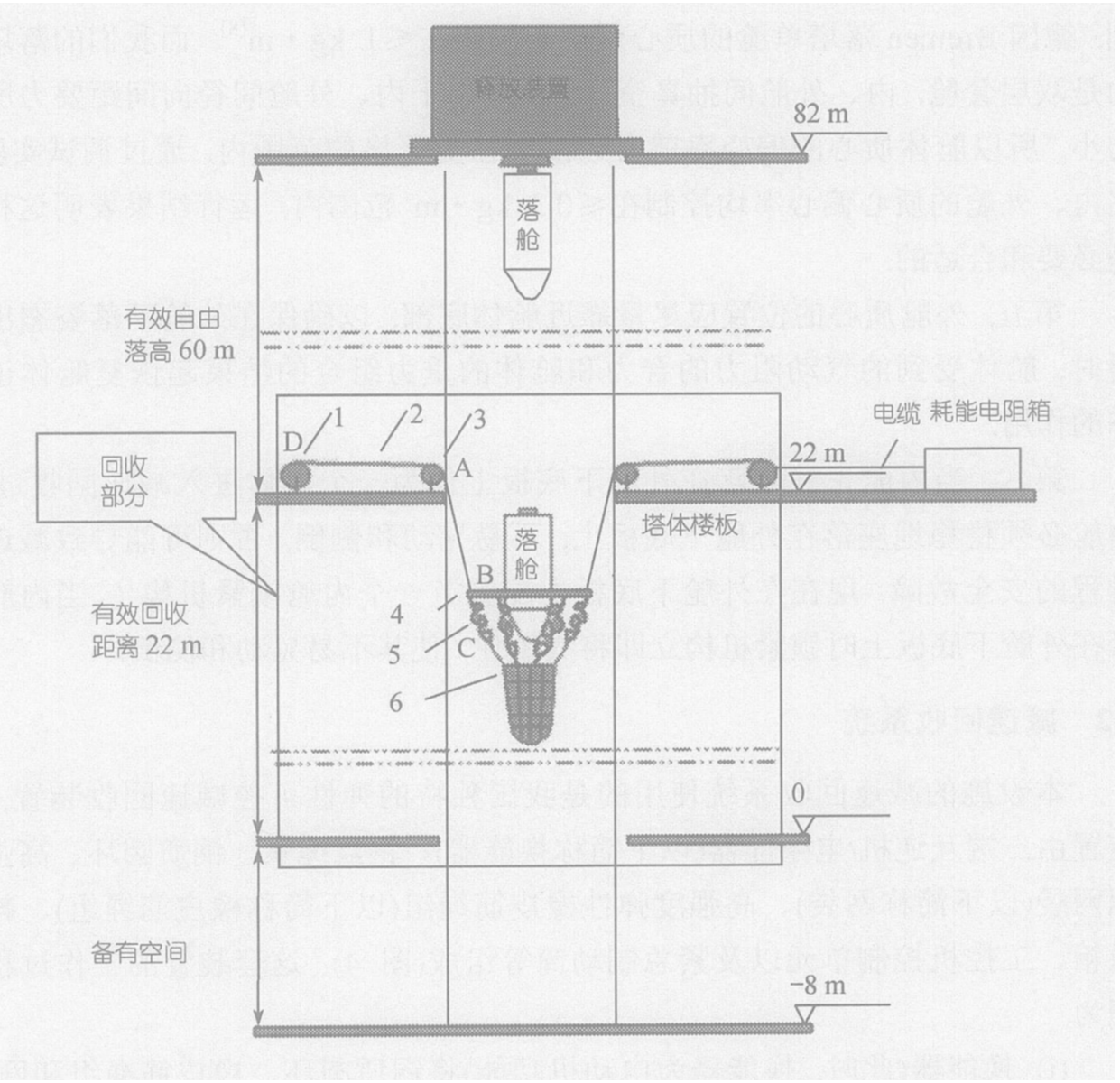

图 4 泛能回收装置示意图

1 示换能器, 2 示钢丝绳索, 3 示导向轮, 4 示钢质圆环, 5 示高强度弹性橡皮筋绳组, 6 示高强度编织网袋

(v) 落舱组件下落速度减小至零, 从固定在地面的坐标上看落舱组件处于瞬 间静止状态. 此时网袋组件仍在下落，换能器还在以较高的转速转动.

(vi) 落舱组件在经过瞬间静止状态后，再次进入自由下落状态. 此时，换能 器一方面将自身的转动动能, 同时也将网袋组件和落舱组件下落时的动能转换 为电能, 由耗能电阻箱消耗掉. 此过程换能器进一步减速.

(vii) 落舱组件再次处于瞬间静止状态.

(viii) 工控机指令整个减速回收系统, 处于刹车状态. 此时, 落舱组件及网 袋组件以极慢的速度缓缓下滑，最后在极限位置完全停止，完成全部减速过程. 图 5 给出加速度计实测曲线可清楚地看出上述减速过程.

这套弹性可控减速回收装置中橡皮筋绳组的弹性系数、橡皮筋绳的长度和耗 能电阻箱的电阻是 3 个独立的参变量. 通过整个系统的空载、部分载荷和满载 


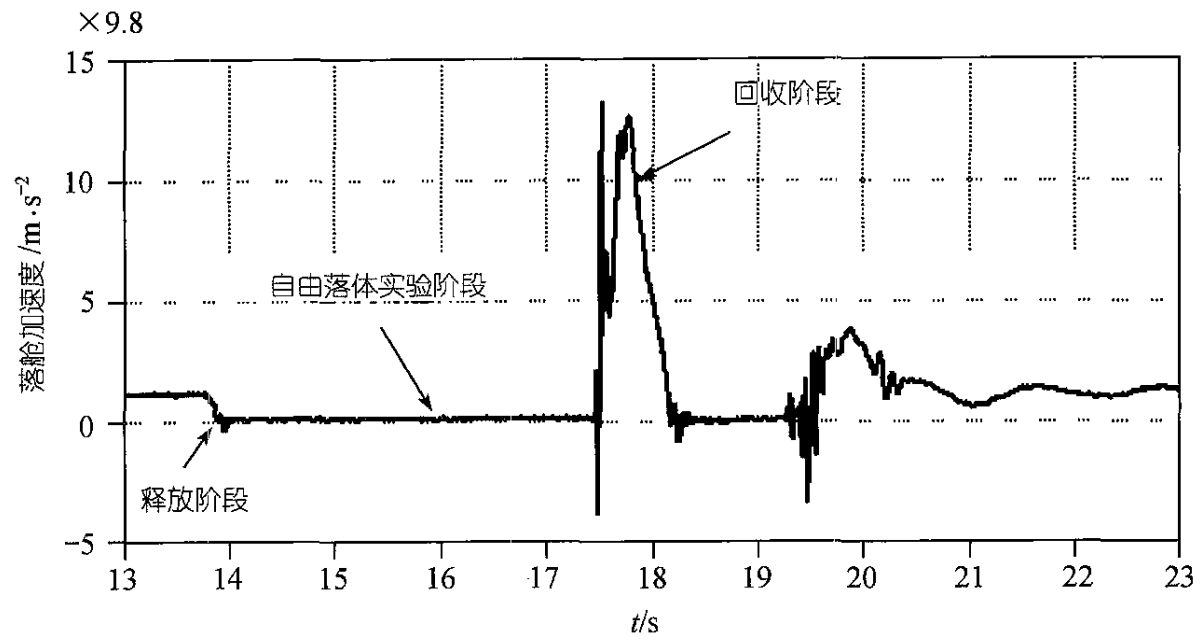

图 5 落舱加速度计实测曲线

荷的运动过程分析计算 ${ }^{1), 2)}$, 初步选定了橡皮筋绳组的弹性系数、橡皮筋绳的长度 和耗能电阻箱的电阻. 由于橡皮筋绳组由 96 条橡皮筋绳组成, 每条橡皮筋绳又 由 250 根橡皮筋组成, 这样只要合理匹配橡皮筋数量、橡皮筋绳数以及橡皮筋绳 的长度就可以调节实验舱减速回收过程受到的弹性阻力. 理论上耗能电阻箱的 电阻是可以连续改变的，以适应实验舱减速过程各种状态. 实际上，在调试过程 中发现只要分档次改变电阻并使其和橡皮筋绳组合理匹配即可满足总体性能对 减速过程的要求。这套减速回收系统必须很好解决的一个关键技术问题是实验 舱在减速过程中不允许“反弹”，也即实验舱由减速前下落速度 $34 \mathrm{~m} / \mathrm{s}$ ，逐步减小 至 $0 \mathrm{~m} / \mathrm{s}$ 后, 不允许实验舱有向上运动的速度. 如果橡皮筋绳组的弹性系数、橡 皮筋绳的长度和耗能电阻的匹配不合适, 橡皮筋绳组就可能将实验舱向上弹起 (反弹). 假如实验舱下落时姿态稍有不正，则“反弹”后会放大实验舱姿态的侧斜. 这样实验舱被 “反弹”至上止点后再次下落时如果姿态侧斜超过允许值就可能导 致回收故障. 调试时, 为了确保安全, 依据分析计算的结果 ${ }^{3)}$ 5), 初步选取了上述 3 个独立参变量的初始值, 然后将实验舱的落差 $\Delta H$ 由 $1,3,5,8,10$ 和 $15 \mathrm{~m}$ 逐步增 加至 $64 \mathrm{~m}$. 每增加一次 $\Delta H$, 调整一次有关参数, 最后妥善地解决了实验舱的“反 弹”问题，而且实验舱的总质量允许在 $600 \sim 630 \mathrm{~kg}$ 之间波动.

1) 中国科学院国家微重力实验室落塔调试组. 在地基落塔上用(发电机+弹性绳+网袋)回收落舱的 运动过程分析计算. 2003

2) 中国科学院国家微重力实验室落塔调试组. 弹性网袋空载制动下滑运动过程的计算. 2003

3) 中国科学院国家微重力实验室落塔调试组. 在地基落塔上用(发电机+弹性绳+网袋)回收落舱的 运动过程分析计算. 2003

4) 中国科学院国家微重力实验室落塔调试组. 弹性网袋空载制动下滑运动过程的计算. 2003

5) 中国科学院国家微重力实验室落塔调试组. 落舱实验系统调试技术总结报告. 2003

www.scichina.com 
与国际上同类设施相比，本设施有下列明显的优越性:

(i) 美国 NASA LeRC 零重力研究设施的减速回收装置是直径 $3.6 \mathrm{~m}$, 深 $6.1 \mathrm{~m}$ 装满疏松的膨化聚乙烯小球的大筒。依靠实验舱表面和膨化聚乙烯小球的摩擦 使实验舱减速，其平均减速过载 $35 g_{0}$, 峰值过载 $65 g_{0}$. 德国 Bremen 落塔采用同 样的减速装置, 其减速筒为直径 $1.6 \mathrm{~m}$, 高 $10 \mathrm{~m}$, 装有 $10 \mathrm{t}$ 疏松的膨化聚乙烯小球 的大筒, 其平均减速过载 $25 g_{0}$, 峰值过载 $<50 g_{0}$. 这种减速过程不能主动控制.

美国 NASA LeRC $2.2 \mathrm{~s}$ 落塔的减速回收装置, 早期(80 世纪 60 年代到 90 年 代初)的技术是实验舱底部有 3 个 $1.9 \mathrm{~m}$ 长的铝质尖雉, 插入 $2.2 \mathrm{~m}$ 深的沙坑内 ${ }^{[10]}$, 冲击很大，减速过载在 40 100 $g_{0}$ 之间. 80 世纪 90 年代中期进行了改进. 现在的 技术状态是气袋回收装置 ${ }^{[3]}$, 实验舱落在充气的气袋上, 气袋受冲击后放气减压 使实验舱减速，采用这种减速技术后，减速过载明显改善，现在只有 $15 \sim 35 g_{0}$, 但是减速过程也未主动控制.

本设施采用的弹性可控减速回收技术，只要合理地匹配橡皮筋绳组的弹性 系数、橡皮筋绳的长度和耗能电阻箱的电阻 3 个独立的参变量, 在工控机(配有专 用软件)的控制下，就能对减速过程实施主动控制. 现在的技术状态是减速过程 近似于半正弦波, 平均减速过载 $8 g_{0}$, 峰值过载 $12 g_{0}$. 这种技术状态还有广阔 的发展空间, 若进一步改进, 还可达到更好的性能. 但是即使是现在的技术状态 也明显优越于膨化聚乙烯小球减速技术和充气气袋减速技术.

(ii) 与日本 JAMIC 落井的磁导轨减速回收技术相比, 减速过载性能基本一 样，但本设施的设备投资要低廉得多.

从综合性能来看, 本设施的减速回收技术水平先进, 是我国独创的原始创新 技术.

\section{3 释放系统}

本设施采用的是电磁释放装置. 由于实验舱是双层套舱，内外舱间抽真空的 结构(内、外舱都必须密封), 就不能使用德国 Bremen 落塔单舱的电动-气动组合 释放机构，也不能使用美国 NASA LeRC 零重力研究设施单舱的悬吊螺栓机构和 $2.2 \mathrm{~s}$ 落塔内外舱间不抽真空(内外舱都不需密封)双层套舱的高强度挂线机构，而 只能参照日本 JAMIC 重型双舱中内舱无直接接触式的电磁机构的原理, 自行研 制一套电磁释放机构.

这套机构由卷扬机组件、83 m 工作面组装平台、电磁吸头组件、外舱被吸 头组件、内舱被吸头组件、外舱机械锁紧/解锁机构、内舱机械锁紧/解锁机构、 零米工作面控制操作台、中心控制室控制操作平台、控制系统，以及 $83 \mathrm{~m}$ 工作 面高空吊篮式操作工作间等组成. 作为释放机构必须妥善解决两个关键技术问 题: 
（i）释放过程释放机构对实验舱的”力”的初始干扰问题.

（ii）释放机构对实验舱初始姿态的影响问题.

由于本装置的释放机构采用的是非机械连接式的电磁吸/放原理, 电磁吸头 与外舱被吸头、内舱被吸头之间 “力”的连系, 不再是“点”连系, 而是“面”连系. 德 国 Bremen 落塔单舱、美国 NASA LeRC 零重力研究设施单舱及 $2.2 \mathrm{~s}$ 落塔内外舱 间不抽真空双层套舱的释放机构均是“点”连系. 而本装置释放机构的“面”联系使 解决上述两个关键技术问题更加困难，具体表现在:

由于加工精度、元、部件组装精度, 静平衡装置精度, 以及实验装置的多样 性, 很难确保:

(i) 电磁吸头组件、外舱组件及内舱组件的质心在其几何中心线上.

(ii) 实验中电磁吸头组件与内外舱组合件对接后在 $83 \mathrm{~m}$ 工作面悬吊待命释 放状态时电磁吸头组件的质心和内外舱组合件的质心在同一重力垂直线上.

(iii) 电磁吸头对实验舱组合件吸力合力作用线通过电磁吸头组件的质心. 这样, 在释放过程中就可能对内、外舱产生“力”的初始干扰, 影响内、外舱在自由 下落时的姿态，从而影响实验装置的实际微重力时间，以及减速回收的安全性.

虽然由于“力”的”面”连系，在技术上存在上述困难，但电磁释放原理却对减 弱释放时的 “力”初始干扰存在有利的影响. 合理的匹配电磁系统的参数可以使释 放过程中, 当吸头电磁线路瞬间断电后, 吸头的吸力不是立即消失, 而是有一个 “缓变”过程, 逐渐消失, 而且磁力的作用可以在一定距离内有效. 这样释放时尽 管实验舱组件开始下落时已经与电磁吸头组件脱离直接接触, 但是电磁吸头仍 然对实验舱 (内舱、外舱) 有吸力, 并且这个吸力随着电磁吸头剩磁吸力的减小和 实验舱与电磁吸头间的空间距离加大而逐渐减小, 直至完全消失. 这样一个渐变 的释放过程就使得内舱中的实验装置在实验时从正常重力状态变成微重力状态, 不是一个阶跃式的突变过程, 而是一个渐变过程, 这样就能有效地减小释放过程 对实验装置的初始干扰.

调试过程中采取了“综合”处理的技术方法 ${ }^{1)}$. 用准确地控制住电磁吸头组件 与实验舱组件连接时 “对中”偏差 $\leqslant 0.25 \mathrm{~mm}$ 和电磁吸头与外、内舱被吸头间“力” 连接链上的关键部位留有 $0.15 \mathrm{~mm}$ 结构间断空间的措施有效地解决了“对中”和 “力”的初始干扰问题, 确保了释放机构安全可靠地工作, 实验舱下落姿态的正直 和减速回收的安全.

\section{3 现状与展望}

最近进行的微重力池沸腾传热实验研究, 获得了稳定的微重力沸腾图像和

1) 中国科学院国家微重力实验室落塔调试组. 落舱实验系统调试技术总结报告. 2003

www.scichina.com 
传热数据. 初步分析表明在壁面过热度较低时, 微重力条件下过冷核态沸腾的传 热效率比常重力约高 $10 \%$ ，而在壁面过热度较高时，微重力条件下过冷双膜态过 渡沸腾的传热效率比常重力约低 $20 \%$. 气泡/膜的行为也有显著变化. 进一步的 实验及对实验数据的详细分析仍在进行中, 部分结果已在 2004 年 5 月 23 27 日 加拿大多伦多 International Symposium on Physical Science in Space/ Spacebound 上发表. 此项实验也为明年将进行的返回式卫星空间搭载实验做了准备, 以验证 空间搭载实验设备的功能和确定空间实验参数.

现在正在进行试验准备工作的有“微重力下层流粉尘火焰传播实验”和“火焰 与流动相互作用实验”. 按计划安排近期将进行试验.

这套实验设施现在每日可进行 2 次实验. 如果等待实验的项目较多，在适当 延长工作时间的情况下，有能力每日进行 4 次实验.

几年来，在所有参与此项目的工作者的共同努力下，百米落塔自由落体实验 设施现已投入正式运行, 承担着我国微重力科学与应用计划中重要的微重力实 验任务, 并将作为一个窗口在微重力科学与应用这一领域内进行广泛的国际合 作. 我国国家微重力实验室百米落塔自由落体实验设施的建成和正式投入使用, 以及其先进、原始独创的技术内涵和性能，显示了我国在这个领域内的实力.

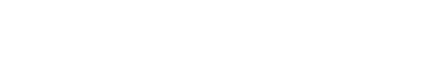

1 Lekan J, Neumann E S, Sotos R G. Capabilities and constraints of NASA's ground-based reduced gravity facilities. In: Second International Microgravity Combustion Workshop, 1993. NASA CP-10113

2 Lekan J. Microgravity Research in NASA Ground-Based Facilities, 1989. AIAA paper 89-0236

3 LeKan J, Gott, D J, Jenkins A J. Users Guide for the 2.2 Second Drop Tower of the NASA Lewis Research Center, 1996. NASA TM 107090

4 JAMIC Japan Microgravity Center, 20. Nishi2-chome, kita-ku, Sapporo 060, Hokkaido, Japan, 1997

5 Japan Microgravity Center User's Guide Japan Microgravity Center (JAMIC). Japan Space Utilization Promotion Center (JSUP), 1991

6 Micro-Gravity Laboratory of Japan (MGLAB), 1990

7 微重力下落实验设施用户指南. 株式会社日本微重力综合研究所, MG-技术资料-002, 1995

8 Drop Tower “Bremen” User Manual. Germany. Version 2.2, 1992

9 ZARM Drop Tower Bremen User Manual. Version 28, 2000

10 John B, Haggard Jr, Thomas H. Cochran, Hydrogen and Hydrocarbon Diffusion Flames in a Weightless Environment. NASA TN D-7165, 1973

SCIENCE IN CHINA Ser. E Engineering \& Materials Science 